\title{
Composite Foaming Agents on the Basis of High-Molecular Natural Surfactants
}

\author{
Fariza Amankeldi *, Zhanar Ospanova * (D) and Kuanyshbek Musabekov \\ Department of analytical, colloid Chemistry and technology of rare elements, al-Farabi Kazakh National \\ University, Almaty 050040, Kazakhstan; zhanar.ospanova@kaznu.kz \\ * Correspondence: fari_z_a@mail.ru (F.A.); zhanospan@mail.ru (Z.O.); \\ Tel.: +7-705-174-46-33 (F.A.); +7-707-698-27-25 (Z.O.)
}

Received: 2 December 2017; Accepted: 4 January 2018; Published: 7 January 2018

\begin{abstract}
Today, naturally occurring foam constituents and surface-active proteins with intriguing structures and functions are being identified from a variety of biological and chemical sources. In this paper we studied the colloid chemical properties of high-molecular natural surfactants such as keratin hydrolyzate (1.5-15\%), gelatin (0.1-1\%), and egg albumin $(0.1-1 \%)$ in a wide concentration range. The foaming ability and foam-stabilizing properties of mixtures of these proteins were established. The high stability of foams obtained from mixtures of surfactants can be explained by the formation of mixed structured layers from the surface-active associates, promoting the thickening of foam films. The ratio of polymer mixtures was optimized (keratin (15\%)-albumin (1\%) (1:1)) to produce high-quality foaming agents. The foam parameters such as surface tension, capillary pressure of the Plateau-Gibbs channels, radii of curvature, critical micelle concentration, and relative viscosity were defined. The high surface activity and foam stability corresponds to a $\mathrm{pH}$ close to the isoelectric state of the proteins. This occurs due to the conformational changes of macromolecules of the protein at the liquid-gas interface, forming particles of colloidal size.
\end{abstract}

Keywords: foam; foam stability; high-molecular natural surfactants; foaming agents; surface tension; capillary pressure

\section{Introduction}

The problem of obtaining highly stable foams from low-cost blowing agents is of interest to many researchers. Foaming agents from natural compounds, plant materials, and animal products have been used by man for food production and for some technological processes for several millennia [1]. Since the keratin-containing raw materials of Kazakhstan and other natural ingredients are readily available, it makes it possible to produce high-molecular foaming agents at an affordable price.

Today, synthetic surfactants-based studies mostly highlight the fundamental aspects of finding the most effective surfactant for better wetting, as well as explore the mechanism, performance enhancement, and reduction of surfactant consumption by the addition of additives or by using mixed surfactant systems [2]. In the case of industrial or domestic applications of these surfactants, especially concerning higher consumption, the disposal of surfactant solutions after the application is a major environmental concern, because synthetic surfactants are not easily biodegradable and sometimes their biodegradable products are also more harmful. So, from the environmental perspective they are not well accepted. Therefore, in the recent years there has been a gradual increasing trend of the application of environmentally friendly, natural surfactants in order to reduce these problems [3].

The purpose of this work is to process the scientific and technical principles of the production of highly stable foam concentrates from natural raw materials, in addition to the determination of their colloid-chemical properties, and the development of an easy and cheap synthesis of surfactants based on natural products. 


\section{Materials and Methods}

Research objects: gelatin (food additive), egg albumin, and keratin hydrolyzate (obtained by the method described in Reference [4], and neutralized with $10 \%$ sulfuric acid solution to $\mathrm{pH} \approx 7$ ).

Foam was generated by the method of shaking from the solutions of surfactants. The initial solution of the surfactant in the amount of $10 \mathrm{~cm}^{3}$ was pipetted into a cylinder with a capacity of $50-100 \mathrm{~cm}^{3}$. The cylinder was shaken for $20 \mathrm{~s}$. After shaking, a stopwatch was switched on, simultaneously marking the volume of the cylinder content $(\mathrm{V})$ and the volume of the remaining liquid $\left(V_{r}\right)$. The time during which the volume of foam decreased twice $\left(t_{1 / 2}\right)$ was registered. The volume and drainage time of foam were determined as an average value of three measurements, between which difference was no more than 10-15\%.

The surface tension of water solutions of high-molecular surfactants was measured by the Wilhelmy plate method, using aluminum plate [5]. All experiments were conducted at room temperature.

The capillary pressure was measured using an apparatus shown Reference [6]. Kinetic measuring of the pressure in the foam channels was carried out until a constant value of $p$, corresponding to the maximum thinning of the film, because foam aging increases the curvature of the foam channels, and thus the pressure in the Plateau-Gibbs channels is reduced. The equilibrium capillary pressure $p_{\sigma}$ was found as difference in pressure $p$ and $p_{0}$.

For the determination of capillary pressure, the Ubbelohde viscometer [6] was used. The measurements were carried out at a constant pressure difference $\Delta p$ at the ends of the capillary of viscometer (the value of $\Delta \mathrm{p}$ was set as directed).

The measurement of each solution was carried out three times (flow time record up to $0.1 \mathrm{~s}$ ), and the average value of flow-time was determined.

\section{Results and Discussions}

\subsection{Foam Stability}

Due to the expansion of the application area of foams there is an interest in exploring new ways to produce foams with high foam stabilizing properties. For this purpose, we studied foaming agents on the basis of protein mixtures KT-Alb (1:1), Alb-Gel (1:1), Gel-KT (1:1), and investigated the stability of the foams of these compositions.

The foam stability results are shown in the graphs below (Figures 1 and 2). These figures show that with an increase of surfactants concentration, the stability of foams also increases. This reveals that the stability of foam directly depends on the concentration and composition of the solution.

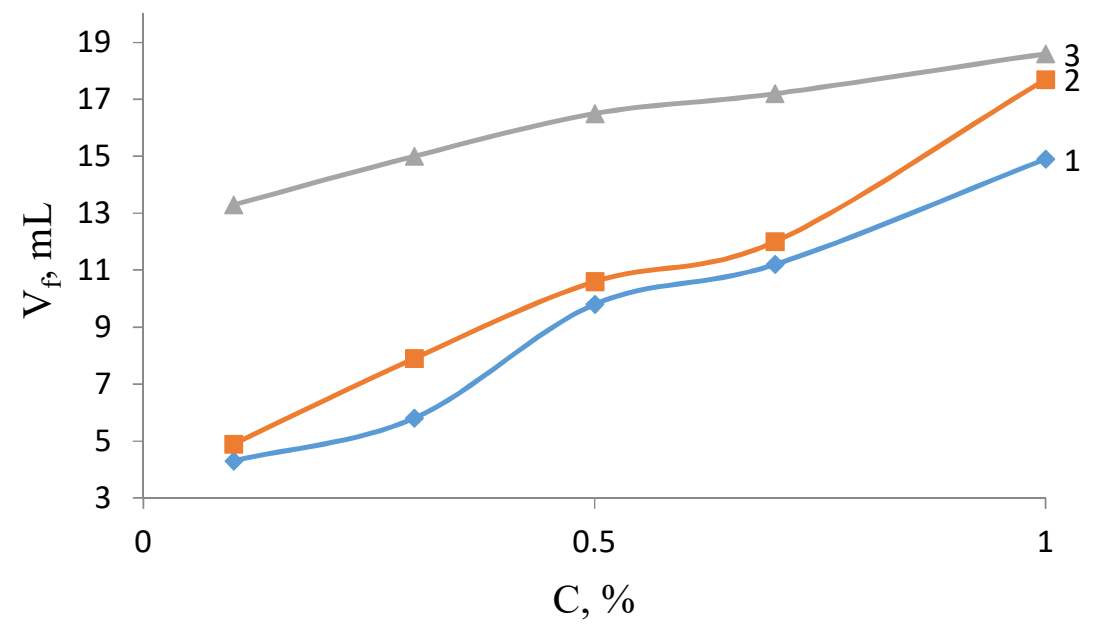

Figure 1. Dependence of the volume of foam $\left(\mathrm{V}_{\mathrm{f}}, \mathrm{mL}\right)$ on the concentration $(\mathrm{C} \%)$ of Gel (1); Alb (2); and a mixture of Gel-Alb in a ratio of 1:1 (3). 


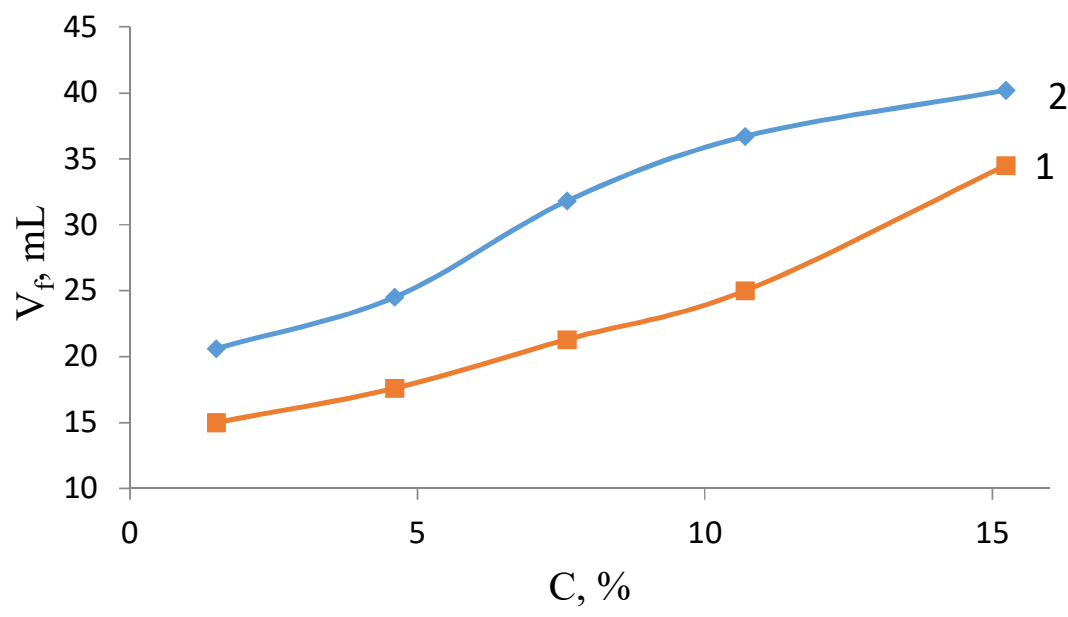

Figure 2. Dependence of the volume of foam $\left(\mathrm{V}_{\mathrm{f}}, \mathrm{mL}\right)$ on the concentration $(\mathrm{C} \%)$ of $\mathrm{KT}(1)$; KT-Alb $(\mathrm{CAlb}=1 \%)(2)$.

From Figure 2, we can observe that among all of the solutions and mixtures, the maximum stable foam forms with a mixture of KT-Alb (1:1). This can be explained by the formation of complexes and associates between polymers, resulting in a change in surface tension and a change in the hydrophilic-lipophilic balance of the protein chain [7]. Increasing foam stability with increasing SAS concentration depends on the adsorption layer.

\subsection{Effect of $\mathrm{pH}$ on the Stability of Foam}

The $\mathrm{pH}$ of solutions plays an important role in the stabilization of the protein foam. The maximum and minimum foaming power depends on the $\mathrm{pH}$. By varying the $\mathrm{pH}$ value, we can influence the foaming intensity of a protein solution.

Proteins are amphoteric polyelectrolytes. They contain ionized basic $\left(-\mathrm{NH}_{3} \mathrm{OH}\right)$ and acidic $(-\mathrm{COOH})$ groups. The dissociation degree of ionized groups of proteins depends on the $\mathrm{pH}$ of the solution. In acidic media, the dissociation of carboxylic groups is suppressed and poly-ions mainly contain positively charged $-\mathrm{RNH}_{3}{ }^{+}$groups. The dissociation degree of amino groups increases in slightly acidic media. As a result, the electrostatic repulsion of $-\mathrm{RNH}_{3}{ }^{+}$groups is also increased, and poly-ampholytes are unfolded into molecular balls. Such conformational transformations of protein molecules occur also in alkaline media. In this case, the dissociation of acidic groups predominates, and poly-ions acquire a negative charge. In weakly alkaline media, the electrostatic interaction of the $-\mathrm{RCOO}^{-}$groups leads to an increase in the effective sizes of the poly-ions. In strongly alkaline media, the number of charged groups in macromolecules decreases because of the formation of ion pairs. Therefore, the protein molecules again fold into denser balls.

The volume and stability of foams reach their maximum at $\mathrm{pH}$ values, corresponding to the isoelectric state for a given protein. In this state they form the most stable films, adsorption layers which exhibit rheological characteristics of solid systems. At the isoelectric point the numbers of interacting ionized basic and acidic groups are equal, and the net charge of poly-ions is zero. As is known [8], the isoelectric points of the SAS are in the range of 4 to 5 . The results of studying surface tension showed that the keratin hydrolyzate exhibits high surface activity in a neutral $\mathrm{pH}$ close to the isoelectric state. The effect of $\mathrm{pH}$ on foam stability is shown in Figure 3. 


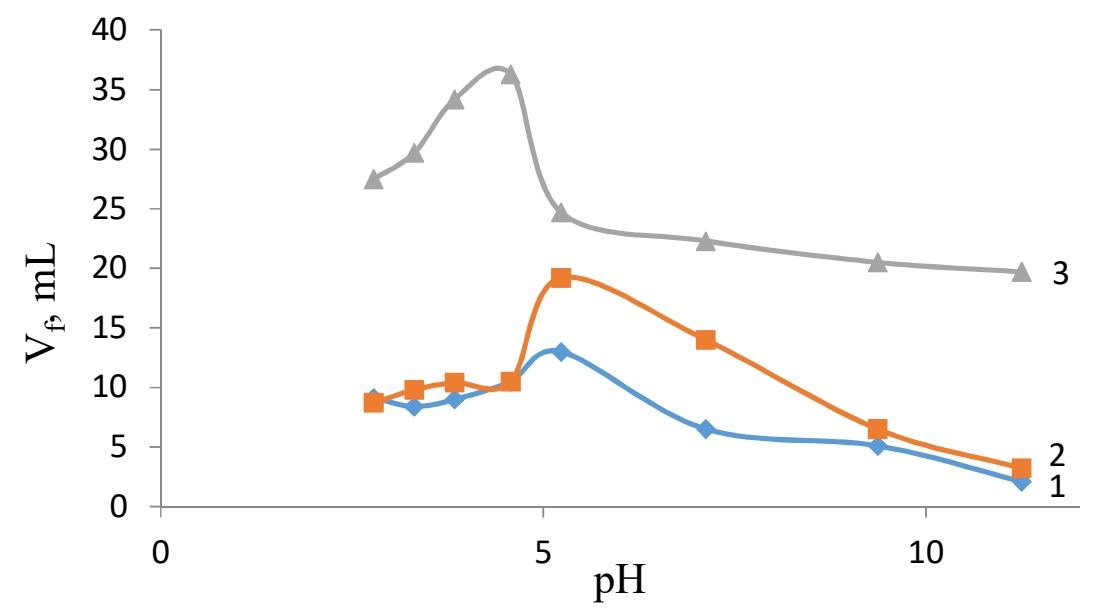

Figure 3. Dependence of volume of foam $\left(\mathrm{V}_{\mathrm{f}}, \mathrm{mL}\right)$ on the $\mathrm{pH}$ of Gel 1\% (1); Alb 1\% (2); and KT 15\% (3).

One reason for the increase in foam stability is that in this state flexible macromolecule proteins coagulate into a ball, forming particles of colloidal dimensions. This facilitates the adsorption of molecules at the interface, lowering the surface tension, and promotes the formation of a strong adsorption layer at the interface-to-air solution. In addition, particles of colloidal size in the formation of foam clog Plateau-Gibbs channels, preventing the expiration of liquid between the films, which is also one of the reasons for increasing the stability of the foam.

\subsection{Capillary Pressure}

The most important property of the foam is reduced (compared to atmospheric) equilibrium capillary pressure in the Plateau-Gibbs channels [9]. Kinetic measurement of pressure in foam channels was performed to obtain a constant value $\Delta \mathrm{P}$, corresponding to the maximum film thinning.

Figure 4 shows that the equilibrium value $\Delta \mathrm{P}$ for foams is obtained from aqueous solutions of the individual surfactants that are higher than the corresponding values of $\Delta \mathrm{P}_{\max }$ for foams from mixtures of SAS.

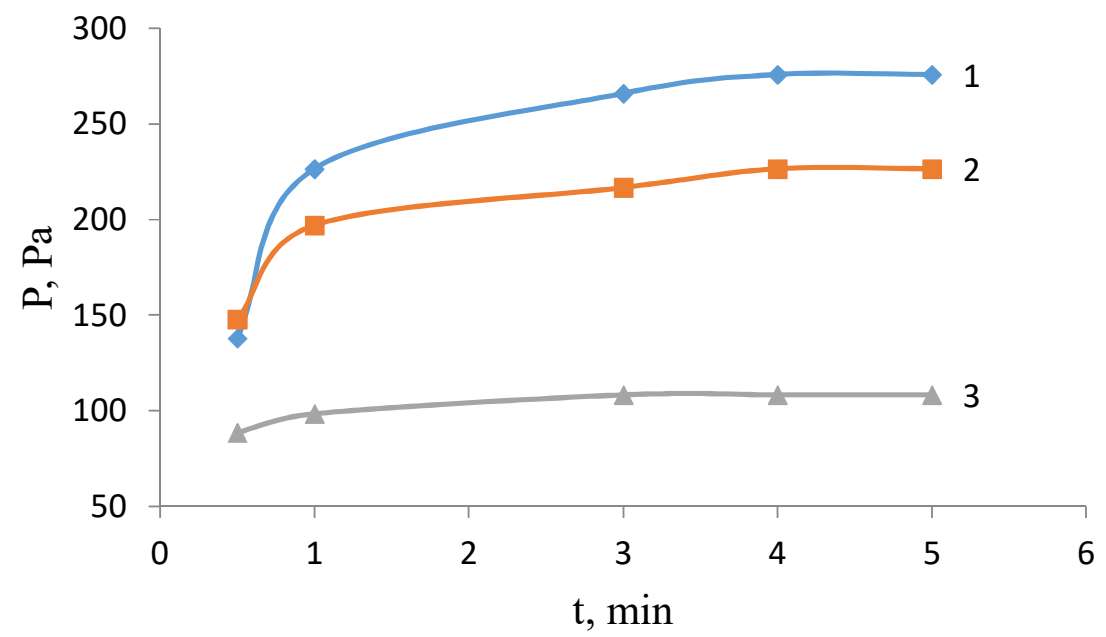

Figure 4. Dependence of capillary pressure (P, Pa) of Alb 1\% (1); KT (15\%)-Alb (1\%) (1:1) (2); Alb (1\%)-Gel (1\%) (1:1) (3) from a given time ( $t$, min).

The values of the equilibrium capillary pressure in foam Plateau-Gibbs channels allow us to judge the equilibrium thickness of the foam films. It can be assumed that the foam films formed from aqueous solutions of the surfactant have reduced thickness in comparison with films of the same 
polymer in the presence of another surfactant. The presence of the adsorbed polymer layer can lead to an increase in the minimum equilibrium film thickness $h_{\min }$. Low values of $\Delta \mathrm{P}$ foam from the binary solution of mixture of surfactants may be associated with an increase in the film thickness due to the formation of mixed adsorption layers of SAS.

The radius of curvature of the Plateau-Gibbs channels was calculated using this formula:

$$
p_{\sigma}=\frac{\sigma}{\mathrm{R}}-\frac{\sigma}{d}
$$

Values of the radius of curvature $(\mathrm{R})$ are shown in Table 1.

Table 1. The radius of curvature of the Plateau-Gibbs channel.

\begin{tabular}{cc}
\hline System & R, m \\
\hline Alb, $1 \%$ & $4.17 \times 10^{-4}$ \\
Alb-KT (1:1) & $3.42 \times 10^{-4}$ \\
Alb-Gel (1:1) & $4.47 \times 10^{-4}$ \\
\hline
\end{tabular}

\subsection{Surface Tension}

As is known, the foaming capacity and stability of foams depend primarily on the surface activity of the components included in the solution. An indirect confirmation of surface activity of the component can be achieved by reducing the surface tension at the liquid-gas interface.

The main factor in the stabilization of foams is the thermodynamic stability factor, which is determined by the specific surface energy (surface tension). The study of the kinetics of lowering the surface tension is of interest due to the fact that the foam is a metastable system, and its parameters change gradually over time. The study of the dynamic characteristics of surface tension allows obtaining information about the process of adsorption, and also serves as an indirect method of studying the structure of adsorbed layers.

In the graphs below (Figures 5 and 6), the surface tension of high-molecular surfactants and their mixtures are investigated.

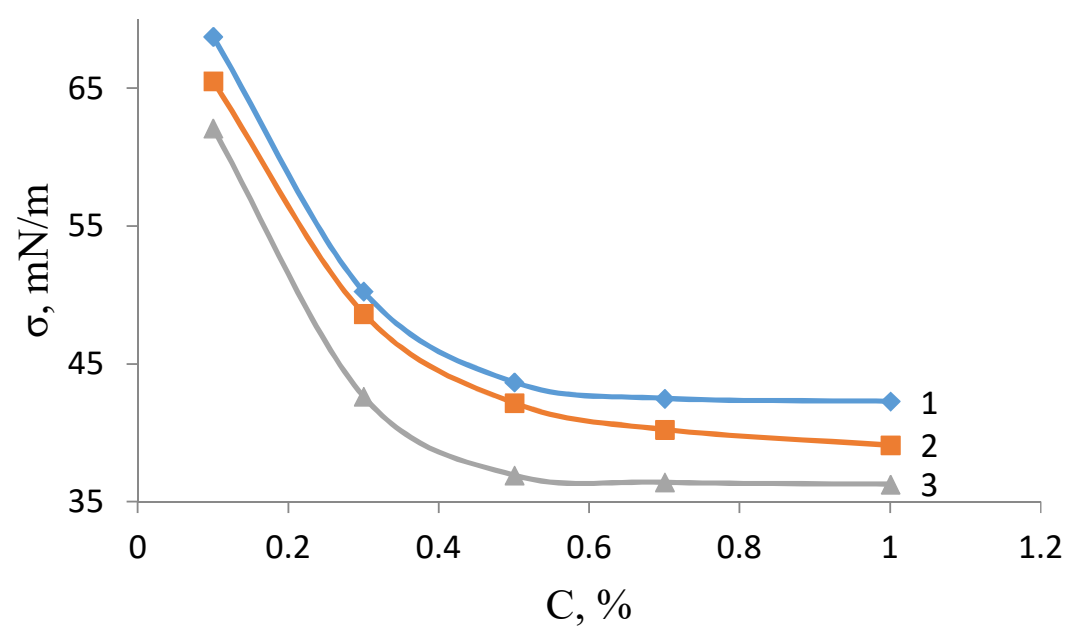

Figure 5. Isotherm of the surface tension of Gel (1); Alb (2); and a mixture of Gel-Alb in a ratio of 1:1 (3).

Figure 5 shows the kinetic curves for reducing surface tension in the concentration range of $0.1-1 \%$. It was found that all studied solutions of foaming agents were capable of reducing the surface tension. Protein hydrolyzates and their surface tension isotherms are poorly understood. It is known that amphiphilic molecules in a solution are concentrated at the interface, thereby reducing the excess free energy of the interface. This spontaneously flowing adsorption process continues until the saturation 
of interface of adsorbed surfactant molecules is reached. Excessive amounts of molecules in the case of micelle-forming surfactant after the saturation of the adsorption layer are combined into aggregates, forming micelles of various structures.

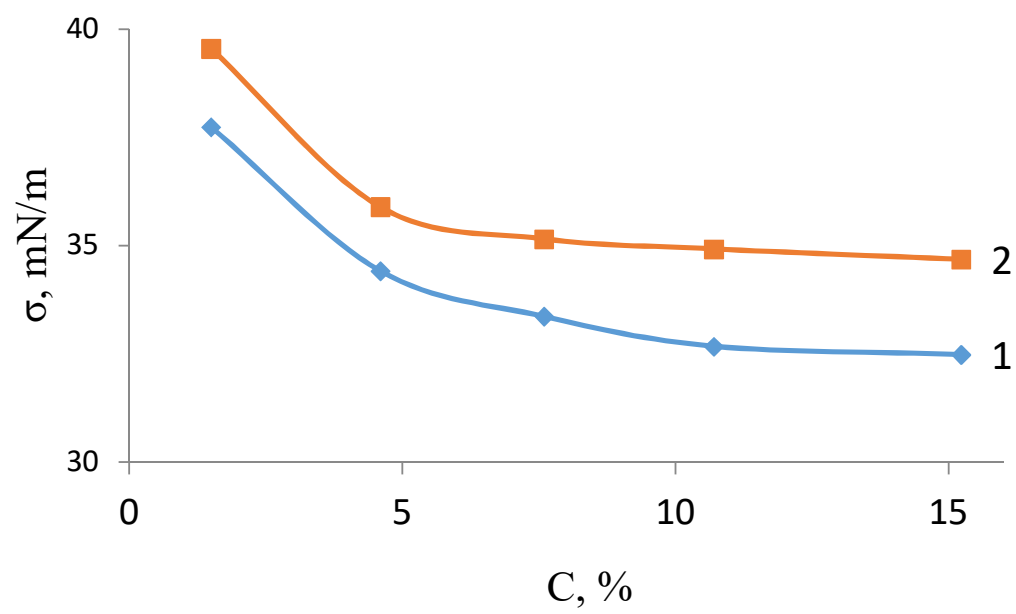

Figure 6. Isotherm of the surface tension of KT (2); and a mixture of KT-Alb (1).

Figure 6 shows that the keratin hydrolyzate has a higher surface activity. The appearance of the fracture on the isotherms of surface tension $\sigma=f(C)$ indicates a possible process of associate formation [10].

On the basis of the isotherms of the surface tension, critical associate concentrations of individual SAS and critical associate concentrations (CACs) of mixtures were calculated. The obtained values of the CACs are shown in Table 2.

Table 2. Critical associate concentrations (CACs) of the analyzed solutions.

\begin{tabular}{cccc}
\hline SAS and Their Mixtures & CAC, $\%$ & SAS and Their Mixtures & CAC, $\%$ \\
\hline Gelatin & 0.56 & Keratin & 5.9 \\
Albumin & 0.53 & - & - \\
Alb-Gel (1:1) & 0.51 & Alb-KT $(1: 1)$ & 5.4 \\
\hline
\end{tabular}

The formation of resistant foam is associated with a reduction in surface tension at the liquid-gas interface. For Gel-Alb, Alb-KT, and KT-Gel mixtures, resistant foam was obtained at concentrations close to the CAC. From Figure 5, it is clear that the equilibrium values of the surface tension of binary mixtures of Gel-Alb are lower than those of the individual components of the isotherms. Reducing the CAC of a solution due to the adsorption of surfactant molecules with their hydrophobic groups causes the formation of aggregates on the expanded polymer chain, which differ in structure and properties compared to the associated individual surfactants. This causes a synergistic effect of the action of the natural surfactant mixture when it is used to reduce the hydrodynamic fluid resistance and the resilience of foams.

The relative viscosity of Alb, Gel, and KT as well as the Alb-Gel (1:1), KT-Alb (1:1), and Gel-KT (1:1) mixtures was calculated using the formula [6]:

$$
\eta_{\mathrm{rel}}=\frac{\eta}{\eta_{0}}=\frac{\tau}{\tau_{0}}
$$

where $\eta$ and $\eta_{0}$ represent the viscosity of the solution and solvent, respectively; $\tau$, $\tau_{0}$ represent the flow time of the solution and the solvent. 
The results are shown in Table 3.

Table 3. Relative viscosities of individual surfactants and their mixtures.

\begin{tabular}{cc}
\hline Surfactants and Their Mixtures & $\eta_{\text {rel }}$ \\
\hline Gel, 1\% & 1.38 \\
KT-Gel (1:1) & 1.46 \\
Alb, 1\% & 1.49 \\
Alb-Gel (1:1) & 1.52 \\
KT, 15\% & 1.57 \\
KT-Alb (1:1) & 1.61 \\
\hline
\end{tabular}

In Table 3, we can see that, compared with individual solutions, the relative viscosity of mixtures is higher. This can be explained by the structural mechanical factor of the stability of foams. Because of the changing structure and due to the formation of protein clews, the flowing of liquid will be complicated and the resultant foam of these mixtures is more stable than that of individual proteins. This may be due to the conformational transformations of macromolecules interacting with the surfactant. A slight increase in the viscosity in the initial part is associated with a small amount of adsorption of surfactant molecules with their hydrophobic groups, which leads to the unfolding of a macromolecule. A further increase in surfactant concentration results in an increase in the viscosity. Increasing the viscosity indicates a strong compaction of associated macromolecules, leading to a decrease in adsorption of molecules at the liquid-air interface.

With the increase of the relative concentration, poly-complexes may form with more unfolded conformations, which lead to a further increase in the viscosity of the solution.

\section{Conclusions}

Foaming compositions from mixtures of KT-Alb (1:1), Gel-KT (1:1), and Alb-Gel (1:1) were found to have higher foaming properties and stability compared with the individual components of KT (15\%), Alb (1\%), Gel (1\%).

The foam-stabilizing action of polymer mixtures was established. The high stability of foams obtained from mixtures of surfactants can be explained by the formation of mixed structured layers from the surface-active associates, promoting the thickening of foam films.

The influence of $\mathrm{pH}$ on the basic characteristics of the obtained foaming agents was investigated. It was revealed that the most stable foams are formed at the isoelectric point of proteins $(\mathrm{pH} \approx 4.5)$.

The capillary pressure of mixtures and individual solutions was determined. This provided information about the thickness of foam films. According to the results of capillary pressure, the radius of curvature was calculated for every composition.

The surface tension of different concentrations of $\mathrm{KT}, \mathrm{Alb}$, and Gel as well as their mixtures (1:1) were determined. We observed that with increasing the concentration of surfactants, the surface tension of solutions decreased. For mixtures of polymers, the thermodynamic factor of stability (synergistic effect of reducing the surface tension) was related to the formation of the surfactant associates.

The relative viscosity of Gel, Alb, KT, Gel-KT (1:1), Alb-Gel (1:1), and KT-Alb (1:1) was calculated. According to the results, we found that the structural-mechanical factor of foam stability was due to the increasing viscosity of the solutions.

These results can be useful for the creation of composite foaming agents for important industries, especially in the food industry, using small amounts of available surfactant polymers. 
Acknowledgments: The work was supported by the grant Project funded by the Ministry of Education and Science of the Republic of Kazakhstan.

Author Contributions: The authors of the article Kuanyshbek Musabekov and Zhanar Ospanova conceived and developed experiments; Fariza Amankeldi conducted experiments; Kuanyshbek Musabekov and Zhanar Ospanova analyzed the data; Kuanyshbek Musabekov provided reagents, materials and tools for analysis; Fariza Amankeldi and Zhanar Ospanova wrote the article.

Conflicts of Interest: The authors declare no conflict of interest.

\section{References}

1. Rahman, P.K.S.M.; Gakpe, E. Production, characterization and applications of bio surfactants. Biotechnology 2008, 7, 360-370. [CrossRef]

2. Foegeding, E.A.; Luck, P.J.; Davis, J.P. Factors determining the physical properties of protein foams. Food Hydrocoll. 2006, 20, 284-292. [CrossRef]

3. Patino, J.M.R.; Sánchez, C.C.; Niño, M.R.R. Implications of interfacial characteristics of food foaming agents in foam formulations. Adv. Colloid Interface Sci. 2008, 140, 95-113. [CrossRef] [PubMed]

4. Ospanova, Z.B.; Musabekov, K.B.; Tokhtarbai, Z.; Isakhov, M.; Koblanova, O.N. Preparation of new foam based on keratin wool. Chem. Bull. Kazakh Natl. Univ. 2012, 3, 206-209. (In Russian) [CrossRef]

5. Rame, E. The interpretation of dynamic contact angles measured by the wilhelmy plate method. J. Colloid Interface Sci. 1997, 185, 245-251. [CrossRef]

6. Nazarov, V.V.; Grodskoy, A.S. Practicum and Exercises of Colloid Chemistry; Academkniga: Moscow, Russia, 2007; pp. 285-289. (In Russian)

7. Von Klitzing, R.; Muller, H.J. Film stability control. Curr. Opin. Colloid Interface Sci. 2002, 7, 42-49. [CrossRef]

8. Mleko, S.; Kristinsson, H.G.; Liang, Y.; Gustaw, W. Rheological properties of foams generated from egg albumin after pH treatment. Food Sci. Technol. 2007, 40, 908-914. [CrossRef]

9. Holmberg, K.; Jonsson, B.; Kronberg, B.; Lindman, B. Surfactants and Polymers in Aqueous Solution; John Wiley \& Sons: Chichester, UK, 2002; pp. 148-159.

10. Cervantes-Martinez, A.; Maldonado, A. Foaming behavior of polymer-surfactant solutions. J. Phys. Condens. Matter 2007, 19, 241-246. [CrossRef] [PubMed]

(C) 2018 by the authors. Licensee MDPI, Basel, Switzerland. This article is an open access article distributed under the terms and conditions of the Creative Commons Attribution (CC BY) license (http:/ / creativecommons.org/licenses/by/4.0/). 